\title{
411.
}

\section{A MEMOIR ON THE THEORY OF RECIPROCAL SURFACES.}

[From the Philosophical Transactions of the Royal Society of London, vol. CLIX. (for the year 1869), pp. 201-229. Received November 12, 1868,-Read January 14, 1869.]

THE present Memoir contains some extensions of Dr Salmon's theory of Reciprocal Surfaces. I wish to put the formulæ on record, in order to be able to refer to them in a "Memoir on Cubic Surfaces," [412], but without at present attempting to completely develope the theory.

\section{Article Nos. 1 to 5. Extension of Salmon's Fundamental Equations.}

1. The notation made use of is that of Salmon's Geometry, [2nd Ed.] pp. 450-459, [but reproduced in the later editions, see Ed. 4. (1882), pp. 580-592], with the additions presently referred to; the significations of all the symbols are explained by way of recapitulation at the end of the Memoir. I remark that my chief addition to Salmon's theory consists in a modification of his fundamental formulæ (A) and (B); these in their original form are

$$
\begin{aligned}
a(n-2) & =\kappa+\rho+2 \sigma, \\
b(n-2) & =\rho+2 \beta+3 \gamma+3 t, \\
c(n-2) & =2 \sigma+4 \beta+\gamma, \\
a(n-2)(n-3) & =2 \delta+3[a c]+2[a b], \\
b(n-2)(n-3) & =4 k+[a b]+3[b c], \\
c(n-2)(n-3) & =6 h+[a c]+2[b c],
\end{aligned}
$$

where

$$
\begin{aligned}
& {[a b]=a b-2 \rho,} \\
& {[a c]=a c-3 \sigma,} \\
& {[b c]=b c-3 \beta-2 \gamma-i .}
\end{aligned}
$$

C. VI. 
2. I take account of conical and biplanar nodes, or, as I call them, cnicnodes, and binodes; of pinch-points $\left({ }^{1}\right)$ on the nodal curve; and of close-points and off-points on the cuspidal curve: viz. I assume that there are

$$
\begin{aligned}
& C, \text { cnicnodes, } \\
& B, \text { binodes, } \\
& j, \text { pinch-points, } \\
& \chi, \text { close-points, } \\
& \theta \text {, off-points, }
\end{aligned}
$$

deferring for the present the explanation of these singularities. The same letters, accented, refer to the reciprocal singularities. Or using "trope" as the reciprocal term to node, these will be

$$
\begin{aligned}
& C^{\prime} \text {, cnictropes, } \\
& B^{\prime} \text {, bitropes, } \\
& j^{\prime}, \text { pinch-planes, } \\
& \chi^{\prime} \text {, close-planes, } \\
& \theta^{\prime} \text {, off-planes; }
\end{aligned}
$$

but these present themselves, not in the equations above referred to, but in the reciprocal equations.

3. The resulting alterations are that we must in the formulæ write $\kappa-B, \delta-C$ in place of $\kappa, \delta$ respectively; and change the formulæ for $c(n-2),[a b],[b c]$, into

respectively.

$$
\begin{gathered}
c(n-2)=2 \sigma+4 \beta+\gamma+\theta, \\
{[a b]=a b-2 \rho-j,} \\
{[a c]=a c-3 \sigma-\chi,}
\end{gathered}
$$

4. Making these changes, and substituting for $[a b],[a c],[b c]$ their values, the formulæ become

$$
\begin{aligned}
& a(n-2)=\kappa-B+\rho+2 \sigma, \\
& b(n-2)=\rho+2 \beta+3 \gamma+3 t, \\
& c(n-2)=2 \sigma+4 \beta+\gamma+\theta, \\
a(n-2)(n-3)=2(\delta-C)+3(a c-3 \sigma-\chi)+2(a b-2 \rho-j), & \\
b(n-2)(n-3)= & 4 k+(a b-2 \rho-j)+3(b c-3 \beta-2 \gamma-i), \\
c(n-2)(n-3)= & 6 h+(a c-3 \sigma-\chi)+2(b c-3 \beta-2 \gamma-i),
\end{aligned}
$$

which replace the original formulæ (A) and (B).

1 This addition to the theory is in fact indicated in Salmon, see the note, p. 445 ; the $i$ there employed, which is of course different from the $i$ of his text, is the $j$ of the present Memoir. 
5. For convenience I annex the remaining equations; viz. these are

$$
\begin{aligned}
& a^{\prime}=n(n-1)-2 b-3 c \\
& \kappa^{\prime}=3 n(n-2)-6 b-8 c \\
& \delta^{\prime}=\frac{1}{2} n(n-2)\left(n^{2}-9\right)-\left(n^{2}-n-6\right)(2 b+3 c)+2 b(b-1)+6 b c+\frac{9}{2} c(c-1)
\end{aligned}
$$

the equations

$$
\begin{aligned}
& q=b^{2}-b-2 k-3 \gamma-6 t, \\
& r=c^{2}-c-2 h-3 \beta,
\end{aligned}
$$

( $q, r$ in place of Salmon's $R, S$ respectively); the equation

$$
a=a^{\prime}
$$

and the corresponding equations, interchanging the accented and unaccented letters, in all 23 equations between the 42 quantities

$$
\begin{aligned}
& n, a, \delta, \kappa ; b, k, t, q, \rho, j ; c, h, r, \sigma, \theta, \chi ; \beta, \gamma, i ; B, C, \\
& n^{\prime}, a^{\prime}, \delta^{\prime}, \kappa^{\prime} ; b^{\prime}, k^{\prime}, t^{\prime}, q^{\prime}, \rho^{\prime}, j^{\prime} ; c^{\prime}, h^{\prime}, r^{\prime}, \sigma^{\prime}, \theta^{\prime}, \chi^{\prime} ; \beta^{\prime}, \gamma^{\prime}, i^{\prime} ; B^{\prime}, C^{\prime} .
\end{aligned}
$$

Article Nos. 6 to 12. Developments.

6. We have

$$
\begin{aligned}
(a-b-c)(n-2) \quad= & (\kappa-B-\theta)-6 \beta-4 \gamma-3 t \\
(a-2 b-3 c)(n-2)(n-3)= & 2(\delta-C) \\
& -8 k-18 h-6(b c-3 \beta-2 \gamma-i) ;
\end{aligned}
$$

and substituting these values of $\delta, \kappa$ in the formula

$$
n^{\prime}=a(a-1)-2 \delta-3 \kappa,
$$

and for $a$ its value, $=n(n-1)-2 b-3 c$, we find

$$
\begin{gathered}
n^{\prime}=n(n-1)^{2}-n(7 b+12 c)+4 b^{2}+8 b+9 c^{2}+15 c \\
-8 k-18 h+18 \beta+12 \gamma+12 i-9 t \\
-2 C-3 B-3 \theta,
\end{gathered}
$$

where the foregoing equations for $a-b-c$ and $a-2 b-3 c$ show clearly the origin of the new terms $-2 C-3 B-3 \theta$; these express that there is in the value of $n^{\prime}$ a reduction $=2$ for each cnicnode, $=3$ for each binode, and $=3$ for each off-point.

7. We have $(n-2)(n-3)=n^{2}-n+(-4 n+6)=a+2 b+3 c+(-4 n+6)$; and making this substitution in the equations which contain $(n-2)(n-3)$, these become

$$
\begin{array}{lr}
a(-4 n+6)=2(\delta-C)-a^{2}-4 \rho-9 \sigma-2 j-3 \chi \\
b(-4 n+6)= & 4 k-2 b^{2}-9 \beta-6 \gamma-3 i+2 \rho-j, \\
c(-4 n+6)= & 6 h-3 c^{2}-6 \beta-4 \gamma-2 i-3 \sigma-\chi
\end{array}
$$


(Salmon's equations (C)); and adding to each equation 4 times the corresponding equation with the factor $(n-2)$, these become

$$
\begin{aligned}
& a^{2}-2 a=2(\delta-C)+4(\kappa-B)-\sigma-2 j-3 \chi, \\
& 2 b^{2}-2 b=4 k-\beta+6 \gamma+12 t-3 i+2 \rho-j, \\
& 3 c^{2}-2 c=6 h+10 \beta+4 \theta-2 i+5 \sigma-\chi .
\end{aligned}
$$

Writing in the first of these $a^{2}-2 a=a(a-1)-a,=n^{\prime}+2 \delta+3 \kappa-a$, and reducing the other two by means of the values of $q, r$, the equations become

$$
\begin{aligned}
n^{\prime}-a & =-2 C-4 B+\kappa-\sigma-2 j-3 \chi \\
2 q+\beta+3 i+j & =2 \rho \\
3 r+c+2 i+\chi & =5 \sigma+\beta+4 \theta
\end{aligned}
$$

(Salmon's equations (D)).

I attend in particular to the first of these, or rather to the reciprocal equation, which will be

$$
\sigma^{\prime}=a-n+\kappa^{\prime}-2 j^{\prime}-3 \chi^{\prime}-2 C^{\prime}-4 B^{\prime},
$$

which, writing therein $a=n(n-1)-2 b-3 c$, and $\kappa=3 n(n-2)-6 b-8 c$, becomes

$$
\sigma^{\prime}=4 n(n-2)-8 b-11 c-2 j^{\prime}-3 \chi^{\prime}-2 C^{\prime}-4 B^{\prime} .
$$

The singularity $\sigma^{\prime}$ is not explicitly defined in Salmon; $\sigma^{\prime}$ is the reciprocal of $\sigma$, and (as such) it denotes the number of common tangent planes of the spinode torse and of the torse generated by the tangent planes along a plane section of the surface; or, what is the same thing, it is the number of the spinode planes which touch the plane section; that is, it is equal to the number of points of intersection of the spinode curve and the plane section; or, finally, $\sigma^{\prime}$ is the order of the spinode curve. The spinode curve is in fact for a surface of the order $n$ without singularities the intersection of the surface by the Hessian surface of the order $4(n-2)$, and is thus a curve of the order $4 n(n-2)$, which agrees with the formula.

8. But the formula shows that there is in the order a reduction $8 b+11 c$ arising from the nodal and cuspidal curves of the surface, or, what is the same thing, that the Hessian surface meets the surface in the nodal curve taken 8 times, and in the cuspidal curve taken 11 times-a result which I had arrived at by other means, and also as appears post, No. 44. The formula shows further that there is a reduction $2 j^{\prime}+3 \chi^{\prime}+2 C^{\prime}+4 B^{\prime}$, or say there are reductions $=2,3,2,4$, for the reciprocals of a pinch-point, a close-point, a cnicnode, and a binode respectively. Geometrically this must signify that the surface and its Hessian partially intersect in certain curves which are not regarded as belonging to the spinode curve. It will at once suggest itself that for the reciprocal of a cnicnode this curve is a conic, and for the reciprocal of a binode it is a line counting 4 times; while for the reciprocal of a pinch-point it is a line counting 2 times, and for the reciprocal of a close-point, a line counting 3 times. 

curve.

9. It is clear that $\rho^{\prime}$ will in like manner denote the order of the node-couple

10. I express in terms of

$$
n, b, c, h, k, \beta, \gamma, j, \theta, \chi, C, B
$$

such quantities and combinations of quantities as can be so expressed. We have

$$
\begin{aligned}
& a=a^{\prime}=n(n-1)-2 b-3 c \text {, } \\
& \kappa^{\prime}=\grave{5} n(n-2)-6 b-8 c \text {, } \\
& \delta^{\prime}=\frac{1}{2} n(n-2)\left(n^{2}-9\right)-\left(n^{2}-n-6\right)(2 b+3 c)+2 b(b-1)+6 b c+\frac{9}{2} c(c-1), \\
& 4 i=12 h+c(5 n-6)-6 c^{2}-5 \gamma+3 \theta-2 \chi, \\
& 24 t=(-8 n+8) b+(15 n-18) c+8 b^{2}-18 c^{2}-2(8 k-18 h)+20 \beta-15 \gamma+4 j+9 \theta+6 \chi \text {, } \\
& q=b^{2}-b-2 k-3 \gamma-6 t, \quad(t \text { suprà }) \text {, } \\
& r=c^{2}-c-2 h-3 \beta \text {, } \\
& 2 \sigma=c(n-2)-(4 \beta+\gamma)-\theta \text {, } \\
& 8 \rho=(16 n-24) b+(-15 n+18) c-8 b^{2}+18 c^{2}+2(8 k-18 h)-9(4 \beta+\gamma)-4 j-9 \theta-6 \chi \text {, } \\
& 8 \kappa=8 n(n-1)(n-2)+b(-32 n+56)+c(-17 n+46)+8 b^{2}-18 c^{2} \\
& -2(8 k-18 h)+17(4 \beta+\gamma)+4 j+17 \theta+6 \chi+8 B, \\
& 2 \delta=n(n-1)(n-2)(n-3)+b\left(-4 n^{2}+20 n-24\right)+c\left(-6 n^{2}+15 n-18\right)+12 b c+18 c^{2} \\
& +(8 k-18 h)-9(4 \beta+\gamma)-9 \theta+2 C, \\
& 8 n^{\prime}=8 n(n-1)^{2}+(-32 n+40) b+(-21 n+30) c+8 b^{2}-18 c^{2} \\
& -2(8 k-18 h)+21(4 \beta+\gamma)-12 j+21 \theta-18 \chi-16 C-24 B, \\
& c^{\prime}=4 n(n-1)(n-2)+(-16 n+28) b+(-10 n+26) c+4 b^{2}-9 c^{2} \\
& -(8 k-18 h)+10(4 \beta+\gamma)-4 j+10 \theta-6 \chi-6 C-8 B, \\
& 2 b^{\prime}=-a+n^{\prime}\left(n^{\prime}-1\right)-3 c^{\prime}, \quad\left(n^{\prime}, c^{\prime} \text { suprà }\right) \text {, } \\
& \sigma^{\prime}+2 j^{\prime}+3 \chi^{\prime}+2 C^{\prime}+4 B^{\prime}=4 n(n-2)-8 b-11 c, \\
& \rho^{\prime}-4 j^{\prime}-6 \chi^{\prime}-4 C^{\prime}-9 B^{\prime}=-11 n(n-2)+a\left(n^{\prime}-2\right)+22 b+30 c, \quad\left(n^{\prime}, a \text { suprà }\right) \text {, } \\
& 2 \sigma^{\prime}+4 \beta^{\prime}+\gamma^{\prime}+\theta^{\prime}=c^{\prime}\left(n^{\prime}-2\right), \quad\left(n^{\prime}, c^{\prime} \text { suprà }\right) \text {, } \\
& 4 k^{\prime}-3\left(i^{\prime}+3 \beta^{\prime}+2 \gamma^{\prime}\right)-2 \rho^{\prime}-j^{\prime}=\left(-4 n^{\prime}+6\right) b^{\prime}+2 b^{\prime 2}, \quad\left(n^{\prime}, b^{\prime} \text { suprà }\right) \text {, } \\
& 6 h-2\left(i^{\prime}+3 \beta^{\prime}+2 \gamma^{\prime}\right)-3 \sigma^{\prime}-\chi^{\prime}=\left(-4 n^{\prime}+6\right) c^{\prime}+3 c^{\prime 2}, \quad\left(n^{\prime}, c^{\prime} \text { suprà }\right) \text {; } \\
& \left.8 k^{\prime}-18 h^{\prime}-4 \rho^{\prime}+9 \sigma^{\prime}-2 j^{\prime}+3 \chi^{\prime}=\left(2 b^{\prime}-3 c^{\prime}\right)\left\{\left(n^{\prime}-2\right)\left(n^{\prime}-3\right)-a\right\}, \quad\left(n^{\prime}, b^{\prime}, c^{\prime}, a \text { suprà }\right)\right\}, \\
& \rho^{\prime}+2 \beta^{\prime}+3 \gamma^{\prime}+3 t^{\prime}=b^{\prime}\left(n^{\prime}-2\right), \quad\left(n^{\prime}, b^{\prime} \text { suprà }\right) \text {, } \\
& 2 q^{\prime}+\beta^{\prime}+3 i^{\prime}+j^{\prime}-2 \rho^{\prime}=0 \text {, } \\
& 3 r^{\prime}+2 i^{\prime}+\chi^{\prime}-5 \sigma^{\prime}-\beta^{\prime}-4 \theta^{\prime}=c^{\prime}, \quad\left(c^{\prime} \text { suprà }\right) \text {, }
\end{aligned}
$$

(twenty-three equations, being a transformation of the original system of twenty-three equations). 
11. Forming the combinations $4 i+6 r, 24 t-8 q+18 r$ (the last of which introduces on the opposite side the term $+48 t$ ), we obtain

$$
\begin{aligned}
4 i+6 r & =c(5 n-12)-5 \gamma-18 \beta+3 \theta-2 \chi, \\
-24 t-8 q+18 r & =-(8 n-16) b+(15 n-36) c-34 \beta+9 \gamma+4 j+9 \theta+6 \chi,
\end{aligned}
$$

equations which are used post, No. 53.

12. I remark that if there be on a surface a right line which is such that the tangent plane is different at different points of the line, the line is said to be scrolar: the section of the surface by any plane through the line contains the line once. But if there is at each point of the line one and the same tangent plane, then the section of the surface by the tangent plane contains the line at least twice; if it contain it twice only, the line is torsal; if three times the line is oscular; and the tangent plane containing the torsal or oscular line may in like manner be termed a torsal or an oscular tangent plane. These epithets, scrolar, torsal and oscular, will be convenient in the sequel.

\section{Article Nos. 13 to 39. Explanation of the New Singularities.}

I proceed to the explanation of the new singularities.

13. The cnicnode, or singularity $C=1$, is an ordinary conical point; instead of the tangent plane we have a proper quadricone.

14. The cnictrope, or reciprocal singularity $C^{\prime}=1$, is also a well known one; it is in fact the conic of plane contact, or say rather the plane of conic contact, viz. the cnictrope is a plane touching a surface, not at a single point, but along a conic.

15. Consider a surface having the cnicnode $C=1$, and the reciprocal surface having the cnictrope $C^{\prime}=1$. There are on the quadricone of the cnicnode six directions of closest contact( $(1)$, and reciprocal thereto we have six tangents of the cnictrope conic, touching it at six points. The plane of the cnictrope meets the surface in the conic twice, and in a residual curve which touches the conic at each of the six points. It would appear that these six contacts are part of the notion of the cnictrope.

16. We may of course have a surface with a conic of plane contact, but such that the residual curve of intersection in the plane of the conic does not touch the conic six times or at all; for instance the general equation of a surface with a conic of plane contact is $P M+V^{2} N=0$, where $P=0$ is a plane, $V=0$ a quadric surface; and here the conic $P=0, V=0$ does not touch the residual curve $P=0, N=0$. The reciprocal surface will in this case have a cnicnode, but there is some special circumstance doing away with the six directions of closest contact which in general belong thereto. I do not further pursue this inquiry.

1 Taking for greater simplicity coordinates $x, y, z, 1$, then for a surface having a cnicnode at the origin, the equation is $U_{2}+U_{3}+\& c .=0$, the suffixes showing the degree in the coordinates; the equation of the quadricone is $U_{2}=0$, and the six directions are given as the lines of intersection of the two cones $U_{2}=0, U_{3}=0$. 
17. For a surface having the cnictrope $C^{\prime}=1$, the Hessian surface passes through the conic, which is thus thrown off from the spinode curve; or there is a reduction $=2$ in the order of the curve, which agrees with a foregoing result.

18. The binode, or singularity $B=1$, is a biplanar node, where instead of the proper quadricone we have two planes; these may be called the biplanes, and their line of intersection, the edge of the binode. The biplanes form a plane-pair.

19. The bitrope, or reciprocal singularity $B^{\prime}=1$, is the plane of point-pair contact; but this needs explanation.

20. Consider a surface having a binode, and the reciprocal surface having a bitrope. We have the bitrope, a plane the reciprocal of the binode; in this plane a line, the reciprocal of the edge; in the line two points, or say a point-pair, the reciprocal of the biplanes: these points may be called the bipoints. There are in each biplane three directions of closest contact; the reeiprocals of these are in the bitrope three directions through each of the two points. The section of the reciprocal surface by the bitrope is made up of the line counting three times (or the line is oscular), and of a curve passing in the three directions (having therefore a triple point) through each of the two bipoints. The bitrope contains thus an oscular line; but it is part of the notion that there are on this line two points each a triple point on the residual curve of intersection.

21. We may however have on a surface an oscular line without upon it two or any triple points of the residual curve of intersection. Such a surface is $M x+N y^{3}=0$; the intersections of the line $x=0, y=0$ with the curve $x=0, N=0$ will be all of them ordinary points. The reciprocal surface will have a binode, but there will be some special circumstance doing away with the existence of the directions of closest contact in the two biplanes respectively. I do not at present pursue the question.

22. For a surface having a bitrope $B^{\prime}=1$, it appears from what precedes, that the oscular line must count 4 times in the intersection of the surface with the Hessian; for only in this way can the reduction 4 in the order of the spinode curve arise.

23. The pinch-point, or singularity $j=1$, is in fact mentioned in Salmon; it is a point on the nodal curve such that the two tangent planes coincide, or say it is a cuspidal point on the nodal curve. If, to fix the ideas, we take the nodal curve to be a complete intersection $P=0, Q=0$, then the equation of the surface is $(A, B, C \gamma P, Q)^{2}=0$ ( $A, B, C$ functions of the coordinates); we have a surface $A C-B^{2}=0$, which may be called the critic surface, intersecting the nodal curve in the points $P=0, Q=0$, $A C-B^{2}=0$, which are the pinch-points thereof; or if there be a cuspidal curve, then such of these points as are not situate on the cuspidal curve are the pinchpoints: see my paper "On a Singularity of Surfaces," Quart. Math. Journ. vol. Ix. (1868) pp. 332-338, [402]. The single tangent plane at the pinch-point meets the surface (see p. 338) in a curve having at the pinch-point a triple point, $=$ cusp +2 nodes, viz. there is a cuspidal branch the tangent to which coincides with that of the nodal curve; and there is a simple branch the tangent to which may be called the cotangent 
at the pinch-point. In the particular case where the nodal curve is a right line the section is the line twice (representing the cuspidal branch), and a residual curve of the order $n-2$, the tangent to which is the cotangent.

24. The pinch-plane, or reciprocal singularity $j^{\prime}=1$, is in fact a torsal plane touching the surface along a line, or meeting it in the line twice and in a residual curve. Let the line and curve meet in a point $P$; for the reason that the section by the plane is the line twice and the residual curve, the section has at $P$ two coincident nodes; that is, the plane is a node-couple plane with two coincident nodes. The plane meets the consecutive node-couple plane in a line $\mu$ passing through $P$ and touching at this point the residual curve. Considering now the reciprocal figure, the reciprocal of the pinch-plane is thus a point of the nodal curve, and is a pinch-point; the tangent plane at the pinch-point is the reciprocal of the point $P$; the tangent to the nodal curve is the reciprocal of the line $\mu$, that is, of the tangent at $P$ to the residual curve; and the cotangent at the pinch-point is the reciprocal of the torsal line.

25. There is in this theory the difficulty that for a surface of the order $n$, the torsal plane meets the residual curve of intersection in $(n-2)$ points $P$, and if each of these be a point on the node-couple curve, then in the reciprocal figure the pinchpoint would be a multiple point on the nodal curve. I apprehend that starting with a pinch-point, a simple point on the nodal curve, we have in the reciprocal figure a pinch-plane or torsal plane as above, but with some speciality in virtue of which only one of the $(n-2)$ points of intersection of the torsal line with the residual plane curve is a point of the node-couple curve of the reciprocal surface. In the case of a pinch-plane or torsal plane of a cubic surface, $n-2$ is $=1$, and the question of multiplicity does not arise.

26. For a surface with a pinch-plane or torsal plane as above $\left(j^{\prime}=1\right)$, the Hessian surface not only passes through the torsal line, but it touches the surface along this line, causing, as already mentioned, a reduction $=2$ in the order of the spinode curve. That the surfaces touch along the line is an important theorem( $\left.{ }^{1}\right)$, and I annex a proof.

27. Let $x=0, y=0$ be the torsal line, $x=0$ being the torsal plane; the equation of the surface therefore is $x \phi+y^{2} \psi=0$; and if $A, B, C, D$ be the first derived functions of $\phi,(a, b, c, d, f, g, h, l, m, n)$ the second derived functions, and if $\left(A^{\prime}, B^{\prime}, C^{\prime}, D^{\prime}\right)$, $\left(a^{\prime}, b^{\prime}, c^{\prime}, d^{\prime}, f^{\prime}, g^{\prime}, h^{\prime}, l^{\prime}, m^{\prime}, n^{\prime}\right)$ refer in like manner to $\psi$, then the equation of the Hessian is

$$
\begin{aligned}
& 0=\mid 2 A+x a+y^{2} a^{\prime} \quad, \quad B+x h+2 y A^{\prime}+y^{2} h^{\prime}, \quad C+x g+y^{2} g^{\prime}, \quad D+x l+y^{2} l^{\prime} \\
& B+x h+2 y A^{\prime}+y^{2} h^{\prime}, \quad x b+2 \psi+4 y B^{\prime}+y^{2} b^{\prime}, \quad x f+2 y C^{\prime}+y^{2} f^{\prime}, \quad x m+2 y D^{\prime}+y^{2} m^{\prime} \\
& C+x g+y^{2} g^{\prime} \quad, x f+2 y C^{\prime}+y^{2} f^{\prime} \quad, x c+y^{2} c^{\prime} \quad, \quad x n+y^{2} n^{\prime} \\
& D+x l+y^{2} l^{\prime} \quad, x m+y^{2} n^{\prime}+2 y D^{\prime} \quad, x n+y^{2} n^{\prime} \quad, \quad x d+y^{2} d^{\prime} ;
\end{aligned}
$$

${ }^{1}$ See Salmon, p. 218, where it is only stated that the Hessian passes through the line. 
and representing this for a moment by

then in the developed equation

$$
\left|\begin{array}{llll}
A, & H, & G, & L \\
H, & B, & F, & M \\
G, & F, & C, & N \\
L, & M, & N, & D
\end{array}\right|=0
$$

$$
\begin{aligned}
& D\left(A B C-A F^{2}-B G^{2}-C H^{2}+2 F G H\right) \\
- & \left(B C-F^{2}, C A-G^{2}, \quad A B-H^{2}, \quad G H-A F, . H F-B G, \quad F G-C H \gamma L, M, N\right)^{2}=0,
\end{aligned}
$$

observing that $C, F, M, N, D$ are of the first order in $x, y$, the only terms of the first order are contained in $B\left(-D G^{2}-C L^{2}+2 N G L\right)$; and since $C, D, N$ are of the first order, we obtain all the terms of the first order by reducing $B, G, L$ to the values $2 \psi, C, D$; viz. the terms of the first order are

$$
2 \psi\left(-C^{2} d x-D^{2} c x+2 C D n x\right),=-2 \psi\left(C^{2} d+D^{2} c-2 C D n\right) x .
$$

Hence the complete equation is of the form

$$
-2 \psi\left(C^{2} d+D^{2} c-2 C D n\right) x+(x, y)^{2}=0,
$$

or, what is the same thing, $x \Phi+y^{2} \Psi=0$; the Hessian has therefore along the line $x=0, y=0$ the same tangent plane $x=0$ as the surface; or it touches the surface along this line; that is, the line counts twice in the intersection of the two surfaces.

28. If instead of the right line we have a plane curve, say if the equation be $x \phi+P^{2} \psi=0$, then the value of the Hessian is $x \Phi+P \Psi=0$ (viz. the second term divides by $P$ only, not by $P^{2}$ ), so that, as before mentioned in regard to a conic of contact, the surface and the Hessian merely cut (but do not touch) along the curve $x=0, P=0$. To show this in the most simple manner take the equation to be $x \phi+\frac{1}{2} P^{2}=0$; let $A^{\prime}, B^{\prime}, C^{\prime}, D^{\prime}$ be the first derived functions of $\phi$, and $(A, B, C, D)$, $(a, b, c, d, f, g, h, l, m, n)$ the first and second derived functions of $P$; then if in the equation of the Hessian we write for greater simplicity $x=0$, - the equation is

$$
\left|\begin{array}{rrrr}
2 A^{\prime}+P a+A^{2}, & B^{\prime}+P h+A B, & C^{\prime}+P g+A C, & D^{\prime}+P l+A D \\
B^{\prime}+P h+A B, & P b+B^{2}, & P f+B C, & P m+B D \\
C^{\prime}+P g+A C, & P f+B C, & P c+C^{2}, & P n+C D \\
D^{\prime}+P l+A D, & P m+B D, & P n+C D, & P d+D^{2}
\end{array}\right|=0 .
$$

The equation contains for example the term

$$
-\left(D^{\prime}+P l+A D\right)^{2}\left\{P^{2}\left(b c-f^{2}\right)+P\left(b C^{2}+c B^{2}-2 f B C\right)\right\}
$$

dividing as it should do by $P$, but not dividing by $P^{2}$; and considering the portion hereof $-D^{\prime 2} P\left(b C^{2}+c B^{2}-2 f B C\right)$, there are no other terms in $D^{\prime 2} P$ which can destroy this, and to make the whole equation divide by $P^{2}$; which proves the required negative.

C. VI. 
29. For the off-point or singularity $\theta=1$; this is a point on the cuspidal curve at which the second derived functions all of them vanish. In further explanation hereof consider a surface $U=0$, and the second polar of an arbitrary point $(\alpha, \beta, \gamma, \delta)$; viz. this is $\left(\alpha \partial_{x}+\beta \partial_{y}+\gamma \partial_{z}+\delta \partial_{w}\right)^{2} U=0$, or say for shortness $\Delta^{2} U=0$, where the coefficients of the powers and products of $(\alpha, \beta, \gamma, \delta)$ are of course the second derived functions of $U$; this equation, when reduced by means of the equations of the cuspidal curve, may acquire a factor $\Lambda$, thus assuming the form $\Lambda(\alpha P+\beta Q+\gamma R+\delta S)^{2}=0$, and if so the intersections of the cuspidal curve with the second polar $(=2 \sigma+\theta$, if, as for simplicity is supposed, there is no nodal curve) will be made up of the intersections of the cuspidal curve with the surface $\Lambda=0$, and of those with the surface $\alpha P+\beta Q+\gamma R+\delta S=0$ each twice; the latter of these, depending on the coordinates $(\alpha, \beta, \gamma, \delta)$ of the arbitrary points, are the points $\sigma$ each twice; the former of them, or intersections of the cuspidal curve with the surface $\Lambda=0$, are the points $\theta$, or off-points of the cuspidal curve. If there is a nodal curve, the only difference is that the off-points are such of the above points as do not lie on the nodal curve.

30. As the most simple instance of the manner in which this singularity may present itself, consider a surface $F P^{2}+G Q^{3}=0$, where the degrees of the functions are $f, p, g, q$, and therefore $n=f+2 p=g+3 q$, if $n$ be the order of the surface. This has a cuspidal curve $P=0, Q=0$ of the order $p q$; the equation $\Delta^{2}\left(F P^{2}+G Q^{3}\right)=0$ of the second polar, when reduced by the equations $P=0, Q=0$ of the cuspidal curve, becomes simply $F(\Delta P)^{2}=0$; and we have thus the off-points $F=0, P=0, Q=0$, consequently $\theta=f p q$.

31. But suppose, as before, the case of a surface $(A, B, C \gamma P, Q)^{2}=0$ having a cuspidal curve $P=0, Q=0$, and there ore $A C-B^{2}$ being $=0$ for $P=0, Q=0$. The equation of the second polar, writing therein $P=0, Q=0$, becomes $(A, B, C \gamma \Delta P, \Delta Q)^{2}=0$, and if for any given surface this assumes the form $\Lambda(M \Delta P+N \Delta Q)^{2}=0$ (observe that $M, N$ may be fractional provided only the $M \Delta P+N \Delta Q$ is integral), then there will be on the cuspidal curve the off-points $\Lambda=0, P=0, Q=0$.

32. An interesting example is afforded by a surface which presents itself in the Memoir on Cubic Surfaces: the surface

$$
\begin{aligned}
& 4 y^{6} \\
- & 4 y^{3} x\left(x^{2}+3 z w\right) \\
+ & z w\left(3 x^{2}+z w\right)^{2}=0
\end{aligned}
$$

has the cuspidal conic $y=0,3 x^{2}+z w=0$, and (as coming under the form $F P^{2}+G Q^{3}=0$ ) has the off-points $z w=0, y=0,3 x^{2}+z w=0$; that is, the points $(x=0, y=0, z=0)$, $(x=0, y=0, w=0)$ each twice; $\theta=4$.

But writing the same equation in the form

where

$$
\left(4,6 x, 8 x^{2}+z w \gamma\left(y^{3}-2 x^{3}, x^{2}-z w\right)^{2}=0,\right.
$$

$$
4 \cdot\left(8 x^{2}+z w\right)-(6 x)^{2}=-4\left(x^{2}-z w\right),
$$


it appears that there are also the three cuspidal conics $y^{3}-2 x^{3}=0, x^{2}-z w=0$. Reducing by means of these two equations, the equation of the second polar is at first obtained in the form

$$
\left(4,6 x, 8 x^{2}+z w \gamma 3 y^{2} \Delta y-6 x^{2} \Delta x, 2 x \Delta x-z \Delta \omega-w \Delta z\right)^{2}=0 ;
$$

but further reducing by the same equations and writing for this purpose $y=\omega x\left(\omega^{3}=2\right)$, the equation becomes

that is

$$
\left(4,6 x, 9 x^{2} \gamma x^{2}\left(3 \omega^{2} \Delta y-6 \Delta x\right), 2 x \Delta x-z \Delta w-w \Delta z^{2}\right)^{2}=0,
$$

$$
x^{2}\left[2 x\left(3 \omega^{2} \Delta y-6 \Delta x\right)+3(2 x \Delta x-z \Delta w-w \Delta z)\right]^{2}=0,
$$

and we have thus the off-points $x^{2}=0, y^{3}-2 x^{3}=0, x^{2}-z w=0$, in fact the beforementioned two points each 6 times; and the complete value of $\theta$ is $\theta=(4+12 \Rightarrow) 16$; viz. the off-points are the points $(x=0, y=0, z=0),(x=0, y=0, w=0)$ each 8 times. On account of this union of points the singularity is really one of a higher order, but equivalent to $\theta=16$.

I am not at present able to explain the off-plane or reciprocal singularity $\theta^{\prime}=1$.

33. As to the close-point or singularity $\chi=1$. I remark that at an ordinary point of the cuspidal curve the section by the tangent plane touches, at the point of contact, the cuspidal curve: the point of contact is on the curve of section a singular point \{in the nature of a triple point, viz. taking the point of contact as origin, the form of the branch in the vicinity thereof is $y^{3}-x^{4}=0$, where $y=0$ is the equation of the tangent to the cuspidal curve\}, such that the point of contact counts 4 times in the intersection of the cuspidal curve with the curve of section. At a close-point the form of the curve of section is altered; viz. the point of contact is here in the nature of a quadruple point with two distinct branches, one of them a triple branch of the form $y^{3}=x^{4}$, but such that the tangent thereof, $y=0$, is not the tangent of the cuspidal curve; the other of them a simple branch, the tangent of which is also distinct from the tangent of the cuspidal branch: the point of contact counts $3+1$ times, that is 4 times, as before, in the intersection of the cuspidal curve and the curve of section. The tangent to the simple branch may conveniently be termed the cotangent at the close-point; that of the other branch the cotriple tangent.

34. We may look at the question differently thus: to fix the ideas, let the cuspidal curve be a complete intersection $P=0, Q=0$; the equation of the surface is $(A, B, C \gamma P, Q)^{2}=0$, where $A C-B^{2}=0$, in virtue of the equations $P=0, Q=0$ of the cuspidal curve, that is, $A C-B^{2}$ is $=M P+N Q$ suppose. We have (as in the investigation regarding the pinch-point) a critic surface $A C-B^{2}=0$, this meets the surface in the cuspidal curve and in a residual curve of intersection; the residual curve by its intersection with the cuspidal curve determines the close-points; the tangent at the close-point is I believe the tangent of the residual curve. Analytically the close-points are given by the equations $P=0, Q=0,(A, B, C \gamma N,-M)^{2}=0$. It is proper to remark that if besides the cuspidal curve there be a nodal curve, only such of the points so determined as do not lie on the nodal curve are the close-points. 
35. I take as an example a surface which is substantially the same as one which presents itself in the Memoir on Cubic Surfaces, viz. the surface $\left(1, w, x y \gamma\left(w^{2}-x y, z\right)^{2}=0\right.$, having the cuspidal conic $w^{2}-x y=0, z=0$. Since in the present case $A C-B^{2}=P$, we have $M=1, N=0$, and the close-points are given by $P=0, Q=0, C=0$; that is, they are the points $(z=0, w=0, x=0)$ and $(z=0, w=0, y=0)$.

36. I first however consider an ordinary point on the cuspidal curve, or conic $w^{2}-x y=0$, $z=0$; the coordinates of any point on the conic are given by $x: y: z: w=1: \theta^{2}: 0: \theta$, where $\theta$ is an arbitrary parameter; we at once find $\theta^{2} x+y-\theta(z+2 w)=0$ for the equation of the tangent plane of the surface or cuspidal tangent plane at the point $\left(1, \theta^{2}, 0, \theta\right)$. Proceeding to find the intersection of this plane with the surface, the elimination of $z$ gives

$$
\left(\theta^{2}, \theta w, x y \gamma w^{2}-x y, \theta^{2} x+y,-2 \theta w\right)^{2}=0,
$$

which is of course the cone, vertex $(x=0, y=0, w=0)$, which passes through the required curve of intersection. In place of the coordinates $x, y$ take the new coordinates $\theta^{2} x-y=2 p$, and $\theta^{2} x+y-2 \theta w=2 q$; we have

and thence

$$
\begin{gathered}
\theta^{2} x=\theta w+p-q, \\
-y=-\theta w+p-q,
\end{gathered}
$$

$$
\begin{array}{ll}
-\theta^{2} x y=p^{2}-(q+\theta w)^{2} & =p^{2}-q^{2}-2 \theta q w-\theta^{2} w^{2}, \\
\theta^{2}\left(w^{2}-x y\right) & =p^{2}-q^{2}-2 \theta q w,
\end{array}
$$

and the equation thus is

$$
\left(\theta^{4}, \theta^{3} w,-p^{2}+q^{2}+2 \theta q w+\theta^{2} w-\chi p^{2}-q^{2}-2 \theta q w, 2 \theta^{2} q\right)=0,
$$

or, what is the same thing,

$$
\left(1, \theta w,-p^{2}+q^{2}+2 \theta q w+\theta^{2} w^{2} \gamma p^{2}-q^{2}-2 \theta q w, 2 q\right)^{2}=0 ;
$$

viz. this is

$$
\left(p^{2}-q^{2}-2 \theta q w\right)^{2}+4 \theta q w\left(p^{2}-q^{2}-2 \theta q w\right)+4 q^{2}\left(-p^{2}+q^{2}+2 \theta q w+\theta^{2} w^{2}\right)=0 ;
$$

or reducing, it is

$$
\left(p^{2}-q^{2}\right)\left(p^{2}-5 q^{2}\right)+8 \theta q^{3} w=0,
$$

the equation of the section in terms of the coordinates $p, q, w$. The equation is satisfied by the values $p=0, q=0$ which belong to the assumed point $\left(1, \theta^{2}, 0, \theta\right)$ of the conic, and in the vicinity of this point we have $p^{4}+8 \theta q^{3} w=0$, which is a triple branch of the form $y^{3}=x^{4}$, the tangent $q=0$ being, it will be observed, the tangent of the conic. But at the close-points, or when $\theta=0$ or $\theta=\infty$, the transformation fails; and these points must be considered separately.

37. At the first of these, viz. the point $z=0, w=0, x=0$, the tangent plane of the surface or cuspidal tangent plane is $x=0$, and this meets the surface in the curve $x=0$, 
$w^{3}(w+2 z)=0$, that is in the line $x=0, w=0$ three times, and in the line $x=0, w+2 z=0$ (that the section consists of right lines is of course a speciality, and it is clear that considering in a more general surface the section as defined by an equation in $(w, z, y)$, the line $w=0$ represents the tangent to a triple branch $w^{3}=z^{4}+\& c$., and the line $w+2 z=0$ the tangent to a simple branch); these lines are each of them, it will be observed, distinct from the tangent to the cuspidal conic, which is $x=0, z=0$. And similarly the tangent plane at the other of the two points is $y=0$, meeting the surface in the curve $y=0, w^{3}(w+2 z)=0$, that is in the line $y=0, w=0$ three times, and in the line $y=0, w+2 z=0$.

38. The close-plane or reciprocal singularity $\chi^{\prime}=1$ is (like the pinch-plane) a torsal plane, meeting the surface in a line twice and in a residual curve; the distinction is that the line and curve have an intersection $P$ lying on the spinode curve; the close-plane is thus a spinode plane; it meets the consecutive spinode plane in a line $\mu$ passing through $P$, and which is, not the tangent of the residual curve. In the reciprocal figure, the reciprocal of the close-plane is on the cuspidal curve, and is a close-point; the reciprocal of the point $P$ is the cuspidal tangent plane; that of the line $\mu$ the tangent of the cuspidal curve; that of the tangent of the residual curve the cotriple tangent; that of the torsal line the cotangent.

39. The torsal line of a close-plane is not a mere torsal line; in fact by what precedes it appears that the surface and the Hessian intersect in this line, counting not twice but three times, and it is thus that the reduction in the order of the spinode curve caused by the close-plane is $=3$.

\section{Article Nos. 40 and 41. Application to a Class of Surfaces.}

40. Consider the surface $F P^{2}+G R^{2} Q^{3}=0$, where $f, p, g, r, q$ being the degrees of the several functions, and $n$ the order of the surface, we have of course $n=f+2 p=g+2 r+3 q$.

There is here a nodal curve, the complete intersection of the two surfaces $P=0, R=0$; hence $b=p r, k=\frac{1}{2} p r(p-1)(r-1),=\frac{1}{2} b(b-p-r+1) ; t=0$; whence $(q)=p r(p+r-2)$. There is also a cuspidal curve the complete intersection of the two surfaces $P=0, Q=0$; hence $c=p q, h=\frac{1}{2} p q(p-1)(q-1),=\frac{1}{2} c(c-p-q+1)$; whence $(r)=p q(p+q-2)$ : I have written for distinction $(q),(r)$, to denote the $q, r$ of the fundamental equations. The two curves intersect in the $p q r$ points $P=0, Q=0, R=0$, which are not stationary points on either curve; that is, $\beta=0, \gamma=0, i=p q r$.

There are on the nodal curve the $j=(f+g) p r$ pinch-points $F=0, P=0, R=0$, and $G=0, P=0, R=0$. There are on the cuspidal curve $\theta=f p q$ off-points $F=0$, $P=0, Q=0$; and there the $g p q$ singular points $G=0, P=0, Q=0$. I find that these last, and also the $\theta$ points each three times, must be considered as close-points, that is, that we have $\chi=(g+3 f) p q$. 
41. We ought then to have

$$
\begin{array}{cl}
b(n-2) & =\rho, \\
c(n-2) & =2 \sigma+\theta ; \\
2(q)+3 i+j & =2 \rho, \\
3(r)+c+2 i+\chi & =5 \sigma+4 \theta
\end{array}
$$

the first two of which give $\rho, \sigma$, and then, substituting their values, the other two equations should become identities. In fact, attending to the values $p r=b, p q=c$, the equations become

$$
\begin{aligned}
& 2 b(p+r-2)+3 b q+b(f+g)=2 b(n-2) \\
& 3 c(p+q-2)+c+2 c r+b(g+3 f)=\frac{5}{2}\{c(n-2)-c f\}+4 c f .
\end{aligned}
$$

The first of these is

$$
2 n=2 p+2 r+3 q+f+g, \quad=(2 p+f)+(2 r+3 q+g),
$$

and the second is

$$
\frac{5}{2} n=3 p+3 q+2 r+g+\frac{3}{2} f,=\frac{3}{2}(2 p+f)+(2 r+3 q+g),
$$

so that the equations are satisfied.

\section{Article No. 42. The Flecnodal Curve.}

42. A point on a surface may be flecnodal, viz. the tangent plane may meet the surface in a curve having at the point a flecnode, that is, a node with an inflexion on one of the branches. Salmon has shown that, for a surface of the order $n$ without singularities, the locus of the flecnodal points, or flecnodal curve, is the complete intersection of the surface by a surface of the order $11 n-24$, which may be called the flecnodal surface, the order of the curve being thus $=n(11 n-24)$. I have succeeded in showing, in a somewhat peculiar way by consideration of a surface of revolution, that if the surface of the order $n$ has a nodal curve of the order $b$, and a cuspidal curve of the order $c$, then that the order of the flecnodal curve is $=n(11 n-24)-22 b-27 c ;$ before giving this investigation, I will by the like principles demonstrate the above-mentioned theorem that the order of the spinode curve is $=4 n(n-2)-8 b-11 c$.

Article Nos. 43 to 47. Surfaces of Revolution, in connexion with the Spinode Curve and the Flecnodal Curve.

43. Consider a plane curve of the order $m$ with $\delta$ nodes and $\kappa$ cusps, and let this be made to revolve about an axis in its own plane, so as to generate a surface of revolution. The complete meridian section is made up of the given curve and of an equal curve situate symmetrically therewith on the other side of the axis; the 
order of the surface is thus $=2 m$. The two curves intersect in $m$ points on the axis and in $m^{2}-m$ points, forming $\frac{1}{2}\left(m^{2}-m\right)$ pairs of points, situate symmetrically on opposite sides of the axes; these last generate $\frac{1}{2}\left(m^{2}-m\right)$ circles, nodal curves on the surface; the nodes generate $\delta$ circles, which are nodal curves on the surface, and the cusps generate $\kappa$ circles, cuspidal curves on the surface. There are $m^{2}-m-2 \delta-3 \kappa$ circles of plane contact corresponding in the plane curve to the tangents perpendicular to the axis. Each of the $m$ points on the axis gives in the surface a pair of (imaginary) lines; and we have thus two sets each of $m$ lines, such that along the lines of each set the surface is touched by an (imaginary) meridian plane; viz. these are the circular planes $x+i y=0, x-i y=0$ passing through the axis. I assume without stopping to show it that these $2 m$ lines are lines not $j^{\prime}$ but $\chi^{\prime}$, that is, that they each reduce the order of the spinode curve by $3\left({ }^{1}\right)$. The inflexions generate $3 m^{2}-6 m-6 \delta-8 \kappa$ circles which constitute the spinode curve on the surface.

44. And we can thus verify that the complete intersection of the surface with the Hessian is made up in accordance with the foregoing theory; viz.

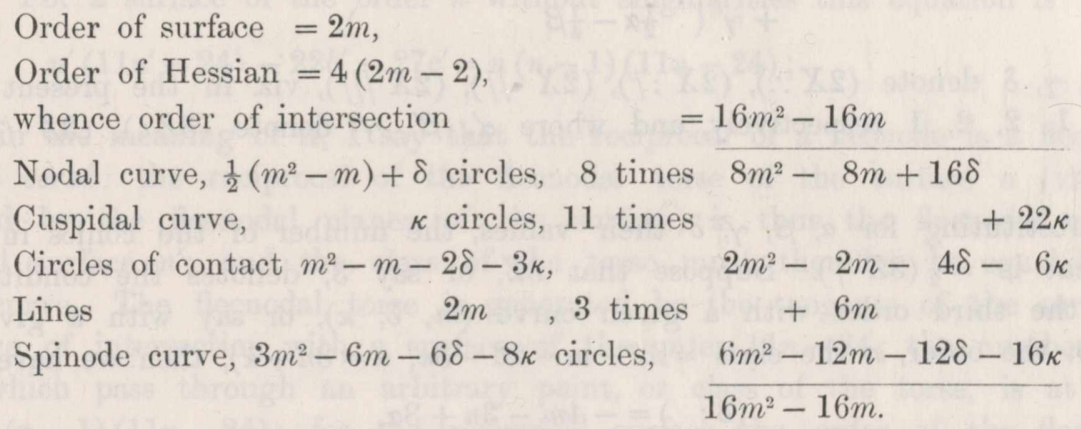

45. We may by a similar reasoning show that the surface and the flecnode surface intersect in the nodal curve taken 22 times, and in the cuspidal curve taken 27 times; and consequently that the order of the residual intersection or flecnodal curve is

$$
=n(11 n-24)-22 b-27 c \text {. }
$$

To effect this, observe that at any point whatever of a quadric surface the tangent plane meets the surface in a pair of lines, that is, in a curve having at the point of contact a node with an inflexion on each branch, or say, a fleflecnode. Imagine in the plane figure a conic having its centre on the axis of rotation and its axis coincident therewith, and the conic having with the curve of the order $m$ a 4-pointic intersection at any point $P$; the point $P$ generates a circle, such that along this circle the surface is osculated by a quadric surface of revolution in such wise that the meridian sections have a four-pointic contact; the circle in question is thus on the surface a fleflecnode circle; and I assume that it counts twice as a flecnode circle. Hence if the number of the points $P$ be $=\theta$, we have on the surface $\theta$ fleflecnode circles, $=2 \theta$ flecnode circles, that is, a flecnode curve of the order $4 \theta$. I wish to show that we have $\theta=5 m^{2}-9 m-10 \delta-12 \kappa$.

1 Observe that the terms in $m$ cannot be got rid of in a different manner, by any alteration of the numbers 8 and 11 to which the present investigation relates. 
46. The problem is as follows: given a curve of the order $m$ with $\delta$ nodes and $\kappa$ cusps; it is required to find the number of the conics, centre on a given line, and an axis coincident in direction with this line, which have with the given curve a 4-pointic intersection, or contact of the third order. This may be solved by means of formulæ contained in my "Memoir on the Curves which satisfy given Conditions," Phil. Trans. vol. clviII. (1868), pp. 75-144; see p. 88; [406].

Taking $x=0$ for the given line, the conic $(a, b, c, f, g, h \chi x, y, 1)^{2}=0$ will have its centre on the given line and an axis coincident therewith, if only $h=0, g=0$; and denoting these two conditions by $2 X$, it is easy to see that we have

$$
(2 X \therefore)=1, \quad(2 X: /)=2, \quad(2 X \cdot / /)=2, \quad(2 X / / /)=1 .
$$

But in general if the conic satisfy any other three conditions $3 Z$, then the number of the conics $(2 X, 3 Z)$ is

$$
\begin{aligned}
= & \alpha^{\prime}\left(-\frac{1}{4} \gamma+\frac{1}{2} \delta\right) \\
+ & \beta^{\prime}\left(-\frac{3}{8} \alpha+\frac{5}{16} \beta+\frac{5}{16} \gamma-\frac{3}{8} \delta\right) \\
+ & \gamma^{\prime}\left(\frac{1}{2} \alpha-\frac{1}{4} \beta\right.
\end{aligned}
$$

where $\alpha, \beta, \gamma, \delta$ denote $(2 X \therefore),(2 X: /),(2 X \cdot / /),(2 X / / /)$, viz. in the present case the values are $1,2,2,1$ respectively, and where $\alpha^{\prime}, \beta^{\prime}, \gamma^{\prime}$ denote $(3 Z:),(3 Z \cdot /),(3 \boldsymbol{Z} / /)$ respectively.

47. Substituting for $\alpha, \beta, \gamma, \delta$ their values, the number of the conics in question is $=\frac{1}{2} \beta^{\prime}$, that is $=\frac{1}{2}(3 Z \cdot /)$. Suppose that $3 Z$, or say 3 , denotes the condition of a contact of the third order with a given curve $(m, \delta, \kappa)$, or say with a given curve $(m, n, \alpha)$ ( $m$ the order, $n$ the class $\left.=m^{2}-m-2 \delta-3 \kappa, \alpha=3 n+\kappa\right)$, then we have

$$
\begin{aligned}
& (3:)=-4 m-3 n+3 \alpha, \\
& (3 \cdot /)=-8 m-8 n+6 \alpha, \\
& (3 / /)=-3 m-4 n+3 \alpha ;
\end{aligned}
$$

and from the second of these the number of the conics in question is $=-4 m-4 n+3 \alpha$, that is, it is $=-4 m+5 n+3 \kappa$, or finally it is $=5 m^{2}-9 m-10 \delta-12 \kappa$.

Hence, assuming that the $2 m$ lines each counts 6 times $\left(^{1}\right)$,

Order of surface $=2 m$

Order of flecnode surface $=11(2 m-24)$ or $22 m-24$

Order of intersection

$$
\begin{gathered}
=\frac{44 m^{2}-48 m}{22 m^{2}-22 m+44 \delta}+54 \kappa \\
2 m^{2}-2 m-4 \delta-6 \kappa \\
\quad+12 m
\end{gathered}
$$

Nodal curve, $\frac{1}{2}\left(m^{2}-m\right)+\delta$ circles, 11 times

Cuspidal curve $\kappa$ circles, 27 times

Circles of contact $m^{2}-m-2 \delta-3 \kappa$,

Lines of contact $2 m \quad, 6$ times

Flecnodal curve, $5 m^{2}-9 m-10 \delta-12 \kappa$ circles each twice $20 m^{2}-36 m-40 \delta-48 \kappa$

$44 m^{2}-48 m$.

1 See foot-note p. 343: the like remark applies to the present terms in $m$, which cannot be got rid of by an alteration of the numbers 22 and 27 to which the investigation relates. 
Article Nos. 48 and 49. The F'lecnodal Torse.

48. Starting from

$$
\begin{aligned}
22 b^{\prime}+27 c^{\prime} & =6\left(6 b^{\prime}+8 c^{\prime}\right)-7\left(2 b^{\prime}+3 c^{\prime}\right) \\
& =6\left(3 n^{\prime 2}-6 n^{\prime}-\kappa\right)-7\left(n^{\prime 2}-n^{\prime}-\delta\right) \\
& =11 n^{\prime 2}-29 n^{\prime}+7-6 \kappa,
\end{aligned}
$$

that is

$$
11 n^{\prime 2}-24 n^{\prime}-22 b^{\prime}-27 c^{\prime}=5 n^{\prime}-7 \delta+6 \kappa,
$$

I find

$$
\begin{aligned}
& n^{\prime}\left(11 n^{\prime}-24\right)-22 b^{\prime}-27 c^{\prime} \\
& =n(n-1)(11 n-24)+b(-59 n+96)+c(-94 n+156)+26 b^{2}+87 c^{2} \\
& \quad-52 k-114 h+141 \beta+94 \gamma+77 i+3 j+4 \chi-15 \theta-45 t-10 C-9 B .
\end{aligned}
$$

49. For a surface of the order $n$ without singularities this equation is

$$
n^{\prime}\left(11 n^{\prime}-24\right)-22 b^{\prime}-27 c^{\prime}=n(n-1)(11 n-24) ;
$$

to explain the meaning of it, I say that the reciprocal of a flecnode is a flecnodal plane, and vice versat: the reciprocal of the flecnodal torse of the surface $n$ (viz. the torse generated by the flecnodal planes of the surface) is thus the flecnodal curve of the reciprocal surface $n^{\prime}$; and the class of the torse must therefore be equal to the order of the curve. The flecnodal torse is generated by the tangents of the surface $n$ along the curve of intersection with a surface of the order $11 n-24$; the number of tangent planes which pass through an arbitrary point, or class of the torse, is at once found to be $n(n-1)(11 n-24)$; for the reciprocal surface the order of the flecnodal curve is by what precedes $n^{\prime}\left(11 n^{\prime}-24\right)-22 b^{\prime}-27 c^{\prime}$; and the equation thus expresses that the order of the curve is equal to the class of the torse.

Article No. 50. The general Surface of the Order $n$ without Singularities.

50. In the general surface of the order $n$ without singularities, we have

$$
\begin{aligned}
n & =n \\
a & =n^{2}-n \\
\delta & =\frac{1}{2} n(n-1)(n-2)(n-3) \\
\kappa & =n(n-1)(n-2) \\
b & =0 \\
k & =0 \\
t & =0 \\
q & =0
\end{aligned}
$$

C. VI. 


$$
\begin{aligned}
& \rho=0, \\
& j=0 \text {, } \\
& c=0 \text {, } \\
& h=0 \text {, } \\
& r=0 \text {, } \\
& \sigma=0 \text {, } \\
& \theta=0 \text {, } \\
& \chi=0 \text {, } \\
& C=0 \text {, } \\
& B=0 \text {, } \\
& \beta=0 \text {, } \\
& \gamma=0 \text {, } \\
& i=0 \text {, } \\
& n^{\prime}=n(n-1)^{2} \text {, } \\
& a^{\prime}=n(n-1) \text {, } \\
& \delta^{\prime}=\frac{1}{2} n(n-2)\left(n^{2}-9\right), \\
& \kappa^{\prime}=3 n(n-2) \text {, } \\
& b^{\prime}=\frac{1}{2} n(n-1)(n-2)\left(n^{3}-n^{2}+n-12\right) \text {, } \\
& k^{\prime}=\frac{1}{8} n(n-2)\left(n^{10}-6 n^{9}+16 n^{8}-54 n^{7}+164 n^{6}-288 n^{5}+547 n^{4}-1058 n^{3}+1068 n^{2}-1214 n+1464\right) \text {, } \\
& t^{\prime}=\frac{1}{6} n(n-2)\left(n^{7}-4 n^{6}+7 n^{5}-45 n^{4}+114 n^{3}-111 n^{2}+548 n-960\right) \text {, } \\
& q^{\prime}=n(n-2)(n-3)\left(n^{2}+2 n-4\right) \text {, } \\
& \rho^{\prime}=n(n-2)\left(n^{3}-n^{2}+n-12\right) \text {, } \\
& j^{\prime}=0 \text {, } \\
& c^{\prime}=4 n(n-1)(n-2) \text {, } \\
& h^{\prime}=\frac{1}{2} n(n-2)\left(16 n^{4}-64 n^{3}+80 n^{2}-108 n+156\right) \text {, } \\
& r^{\prime}=2 n(n-2)(3 n-4) \text {, } \\
& \sigma^{\prime}=4 n(n-2) \text {, } \\
& \theta^{\prime}=0 \text {, } \\
& \chi^{\prime}=0 \text {, } \\
& C^{\prime}=0 \text {, } \\
& B^{\prime}=0 \text {, } \\
& \beta^{\prime}=2 n(n-2)(11 n-24), \\
& \gamma^{\prime}=4 n(n-2)(n-3)\left(n^{3}+3 n-16\right) \text {, } \\
& i^{\prime}=0 \text {. }
\end{aligned}
$$


Article Nos. 51 to 64 . Investigation of Formula for $\beta^{\prime}$.

51. The value $\beta^{\prime}=2 n(n-2)(11 n-24)$ for a surface without singularities was obtained by Salmon by independent geometrical considerations, viz. he obtains

$$
2 \beta^{\prime}=4 n(n-2)(11 n-24)
$$

as the number of intersections of the spinode curve (order $=4 n(n-2))$ by the flecnode surface of the order $11 n-24$.

52. The value of $\beta^{\prime}$ must be obtainable in the case of a surface with singularities, and $\mathrm{I}$ have been led to conclude that we have

$$
\begin{aligned}
\beta^{\prime}= & 2 n(n-2)(11 n-24) \\
& -(110 n-272) b+44 q \\
& -(116 n-303) c+\frac{63}{2} r \\
& +\frac{691}{2} \beta+248 \gamma+198 t \\
& + \text { linear function }\left(i, j, \theta, \chi, C, B, i^{\prime}, j^{\prime}, \theta^{\prime}, \chi^{\prime}, C^{\prime}, B^{\prime}\right),
\end{aligned}
$$

but I have not yet completely determined the coefficients of the linear function. The reciprocal formula in the case of a surface of the order $n$ without singularities, $i, j, \theta, \chi, C, B, i^{\prime}, j^{\prime}, \theta^{\prime}, \chi^{\prime}, C^{\prime}, B^{\prime}$ then all vanishing, is the identity

$$
\begin{aligned}
0= & 2 n^{\prime}\left(n^{\prime}-2\right)\left(11 n^{\prime}-24\right) \\
& -\left(110 n^{\prime}-272\right) b^{\prime}+44 q^{\prime} \\
& -\left(116 n^{\prime}-303\right) c^{\prime}+\frac{63}{2} r^{\prime} \\
& +\frac{691}{2} \beta^{\prime}+248 \gamma^{\prime}+198 t^{\prime}
\end{aligned}
$$

$\left(n^{\prime}, b^{\prime}, q^{\prime}, c^{\prime}, r^{\prime}, \beta^{\prime}, \gamma^{\prime}, t^{\prime}\right.$ having the values in the foregoing Table). It was by assuming for $\beta$ an expression of the above form but with indeterminate coefficients, and then determining these in such wise that the reciprocal equation should be an identity, that the foregoing formula for $\beta^{\prime}$ was arrived at.

53. I assume

$$
\begin{aligned}
\beta^{\prime}= & 2 n(n-2)(11 n-24) \\
& -b(A n-B)+C q \\
& -c(D n-F)+F r \\
& -G \beta-H_{\gamma}-I t \\
& + \text { linear function }\left(i, j, \theta, \chi, C, B, i^{\prime}, j^{\prime}, \theta^{\prime}, \chi^{\prime}, C^{\prime}, B^{\prime}\right),
\end{aligned}
$$

where it is to be remarked that, in virtue of the equations obtained No. 11, two of the coefficients of this form are really arbitrary: I cannot recall the considerations which led me to write $D=116, E=303$. 
54. Forming the reciprocal equation

$$
\begin{aligned}
\beta= & 2 n^{\prime}\left(n^{\prime}-2\right)\left(11 n^{\prime}-24\right) \\
& -b^{\prime}\left(A n^{\prime}-B\right)+C^{\prime} q \\
& -c^{\prime}\left(D n^{\prime}-E\right)+F^{\prime} r \\
& -G \beta^{\prime}-H \gamma^{\prime}-I t^{\prime} \\
& + \text { linear function }\left(i^{\prime}, j^{\prime}, \theta^{\prime}, \chi^{\prime}, C^{\prime}, B^{\prime}, i, j, \theta, \chi, C, B\right),
\end{aligned}
$$

and substituting herein the values which belong to the surface of the order $n$ without singularities, we should have identically

$$
\begin{aligned}
0= & 2 n(n-1)^{2}(n-2)\left(n^{2}+1\right)\left(11 n^{3}-22 n^{2}+11 n-24\right) \\
& -\frac{1}{2} n(n-1)(n-2)\left(n^{3}-n^{2}+n-12\right)\left[A n(n-1)^{2}-B\right] \\
& +n(n-2)(n-3)\left(n^{2}+2 n-4\right) C \\
& -4 n(n-1)(n-2)\left[D n(n-1)^{2}-E\right] \\
& +2 n(n-2)(3 n-4) F \\
& -2 n(n-2)(11 n-24) G \\
& -4 n(n-2)(n-3)\left(n^{3}+3 n-16\right) H \\
& -\frac{1}{6} n(n-2)\left(n^{7}-4 n^{6}+7 n^{5}-45 n^{4}+114 n^{3}-111 n^{2}+548 n-960\right) I
\end{aligned}
$$

or dividing the whole by $n(n-2)$, this is

$$
\begin{aligned}
& 0=2(n-1)^{2}\left(n^{2}+1\right)\left(11 n^{2}-22 n^{2}+11 n-24\right) \\
& -\frac{1}{2}(n-1)\left(n^{3}-n^{2}+n-12\right)\left[A n(n-1)^{2}-B\right] \\
& +(n-3)\left(n^{2}+2 n-4\right) C \\
& -4(n-1)\left[D n(n-1)^{2}-E\right] \\
& +2(3 n-4) F \\
& -2(11 n-24) G \\
& -4(n-3)\left(n^{3}+3 n-16\right) H \\
& -\frac{1}{6}\left(n^{7}-4 n^{6}+7 n^{5}-45 n^{4}+114 n^{3}-111 n^{2}+548 n-960\right) I .
\end{aligned}
$$

55. And then, expanding in powers of $n$ and equating to zero the coefficients of the several powers $n^{7}, \ldots n^{0}$, we obtain

$$
\begin{aligned}
& 22-88+154-224+250-184+118-48 \\
& -\frac{1}{2} A+2 A-\frac{7}{2} A+\frac{19}{2} A-20 A+\frac{37}{2} A-6 A \\
& +\frac{1}{2} B-B+B-\frac{13}{2} B+6 B \\
& +C-C-10 C+12 C \\
& -4 D+12 D-12 D+4 D \\
& +4 E-4 E \\
& +6 F^{\prime}-8 F^{\prime} \\
& -22 G+48 G \\
& \begin{array}{cccccccc} 
& & -4 H & +12 H & -12 H & +100 H & -192 H \\
-\frac{1}{6} I & +\frac{2}{3} I & -\frac{7}{6} I & +\frac{15}{2} I & -19 I & +\frac{37}{2} I & -\frac{274}{3} I & +160 I \\
\| & \| & \| & \| & \| & \| & \| & \| \\
0 & 0 & 0 & 0 & 0 & 0 & 0 & 0
\end{array}
\end{aligned}
$$


viz. the equations are read vertically downwards. The first, second, and third equations, and the sum of the fourth and fifth, all give the same relation, $132-3 A-I=0$; there are consequently, inclusive of this, five independent relations. By combining the equations so as to simplify the numbers, I find these to be

$$
\begin{aligned}
& 3 A+I-132=0, \\
& 4 A-B-2 C-80=0, \\
& 7 A-B+2 E+2 F+2 G-476=0, \\
& 26 A-B+8 D+8 H \quad-1532=0, \\
& 6 A-E-2 F+12 G-48 H+40 I-132=0 .
\end{aligned}
$$

56. I found, as presently mentioned, $A=110, B=272, C=44$; values which satisfy (as they should do) the second equation; and then assuming $D=116$ and $E=303$, we have $F=\frac{63}{2}, G=-\frac{691}{2}, H=-248, I=-198$; and the formula is

$$
\begin{aligned}
\beta^{\prime}= & 2 n(n-2)(11 n-24) \\
& -(110 n-272) b+44 q \\
& -(116 n-303) c+\frac{63}{2} r \\
& +\frac{691}{2} \beta+248 \gamma+198 t \\
& + \text { linear function }\left(i, j, \theta, \chi, C, B, i^{\prime}, j^{\prime}, \theta^{\prime}, \chi^{\prime}, C^{\prime}, B^{\prime}\right),
\end{aligned}
$$

the process not enabling the determination of the coefficients of the linear function.

57. The values of $A, B, C$ were found from the general theorem that if three surfaces of the orders $\mu, \nu, \rho$ respectively intersect in a curve of the order $m$ and class $r$ which is $\alpha$-tuple on $\mu, \beta$-tuple on $\nu$, and $\gamma$-tuple on $\rho$, then the number of the points of intersection of the three surfaces is

$$
=\mu \nu \rho-m(\beta \gamma \mu+\gamma \alpha \nu+\alpha \beta \rho-2 \alpha \beta \gamma)+\alpha \beta \gamma r .
$$

Apply this to the case of a surface of the order $n$ with a nodal curve of the order $b$ and class $q$, intersecting the Hessian and flecnodal surfaces, we have

Order. Passing through $(b, q)$, times

$\begin{array}{lcr}\text { Surface } & n & 2 \\ \text { Hessian } & 4 n-8 & 4 \\ \text { Flecnodal } & 11 n-24 & 11\end{array}$

whence number of intersections is

$$
\begin{aligned}
=4 n(n-2)(11 n-24) & -b\{n .4 .11+(4 n-8) 11.2+(11 n-24) 2.4-2.2 .4 .11\} \\
+ & 2.4 .11 q
\end{aligned}
$$

that is

$$
=4 n(n-2)(11 n-24)-(220 n-544) b-88 q ;
$$


and the value of $\beta^{\prime}$ is one half of this,

$$
=2 n(n-2)(11 n-24)-(110 n-272) b+44 q .
$$

I have not succeeded in applying the like considerations to the cuspidal curve.

58. As regards the general theorem, we know (Salmon, p. 274) that if two surfaces of the orders $\mu, \nu$ partially intersect in a curve of the order $m$ and class $r$, and besides in a curve of the order $m^{\prime}$, then the curves $m, m^{\prime}$ meet in $m(\mu+\nu-2)-r$ points.

Suppose that the curve $m$ is $\alpha$-tuple on the surface $\mu$; then to find the number $I$ of the intersections of the curves $m$ and $m^{\prime}$, we may imagine through $m$ a surface of the order $\rho$; the surfaces $\mu, \nu$ intersect in the curve $m \alpha$ times, and in a residual curve of the order $\mu \nu-m \alpha$, this last meets the surface $\rho$ in $\rho(\mu \nu-m \alpha)$ points, and thence the three surfaces meet in $\mu \nu \rho-m \alpha \rho-I$ points. But since $m$ is a simple curve on each of the surfaces $\nu, \rho$, the three surfaces meet in $\mu(\nu \rho-m)-\alpha[m(\nu+\rho-2)-r]$ points, whence equating the two values

$$
I=m(\mu+\alpha \nu-2 \alpha)-\alpha r .
$$

Next, let the curve $m$ be $\alpha$-tuple on the surface $\mu, \beta$-tuple on the surface $\nu$. Considering the new surface $\rho$ through $m$, then $\mu, \nu$ intersect in the curve $m \alpha \beta$ times, and in a residual curve of the order $\mu \nu-m \alpha \beta$; this last meets the surface $\rho$ in $\rho(\mu \nu-m \alpha \beta)$ points; whence the three surfaces meet in $\rho(\mu \nu-m \alpha \beta)-I$ points. But the curve $m$ being a $\beta$-tuple curve on $\nu$, and a simple curve on $\rho$, these meet in the curve $m \beta$ times and in a residual curve of the order $\nu \rho-\beta m$, whence the three surfaces meet in

$$
\mu(\nu \rho-\beta m)-\alpha[m(\nu+\beta \rho-2 \beta)-\beta r]
$$

points; and equating the two values, we have

$$
I=m(\beta \mu+\alpha \nu-2 \alpha \beta)-2 \alpha \beta r .
$$

Lastly, if the curve $m$ be $\gamma$-tuple on $\rho$, then the surfaces $\mu, \rho$ meet in $m$ ar times and in a residual curve of the order $\mu \rho-m \alpha \gamma$; this last meets $\nu$ in

$$
\nu(\mu \rho-\alpha \gamma m)-\beta[m(\gamma \mu+\alpha \rho-2 \alpha \gamma)-\alpha \gamma r]
$$

points, that is, the number of points of intersection of the three surfaces is

$$
=\mu \nu \rho-m(\beta \gamma \mu+\gamma \alpha \nu+\alpha \beta \rho-2 \alpha \beta \gamma)+\alpha \beta \gamma r .
$$

59. I represent the complete value of $\beta^{\prime}$ by

$$
\begin{aligned}
\beta^{\prime}= & 2 n(11 n-24) \\
& -(110 n-272) b+44 q \\
& -(116 n-303) c+\frac{63}{2} r \\
& +\frac{69}{2} \beta+248 \gamma+198 t \\
& -h C-g B-x i-\lambda j-\mu \chi-\nu \theta \\
& -h^{\prime} C^{\prime}-g^{\prime} B^{\prime}-x^{\prime} i^{\prime}-\lambda^{\prime} j^{\prime}-\mu^{\prime} \chi^{\prime}-\nu^{\prime} \theta^{\prime},
\end{aligned}
$$


and (observing that the Table of Singularities in my Memoir on Cubic Surfaces was obtained without the aid of the formula now in question) I endeavour ky means of the results therein contained to find the values of the unknown coefficients $h, g, x, \lambda, \mu, \nu$, $h^{\prime}, g^{\prime}, x^{\prime}, \lambda^{\prime}, \mu^{\prime}, \nu^{\prime}$.

60. For a cubic surface $n=3$, and for a cubic surface without singular lines (in fact for all the cases except the cubic scrolls XXII and XXIII), the formula is

$$
\beta^{\prime}=54-h C-g B-\lambda^{\prime} j^{\prime}-\mu^{\prime} \chi^{\prime}-\nu^{\prime} \theta^{\prime}-h^{\prime} C^{\prime}-g^{\prime} B^{\prime}
$$

and applying this to the several cases of cubic surfaces as grouped together in the Table, and referred to by the affixed roman numbers, the resulting equations are

$$
\begin{aligned}
54 & =54 \\
30 & =54-h, \\
18 & =54-g-16 \nu^{\prime}, \\
13 & =54-2 h-\lambda^{\prime}, \\
6 & =54-h-g-\mu^{\prime}-8 \nu^{\prime}, \\
3 & =54-3 h-3 \lambda^{\prime} \\
0 & =54-2 g-16 \nu^{\prime}-g^{\prime} \\
1 & =54-2 h-g-\lambda^{\prime}-2 \mu^{\prime}, \\
0 & =54-4 h-6 \lambda^{\prime}, \\
0 & =54-h-2 g-2 \mu^{\prime}-g^{\prime}, \\
0 & =54-3 g-3 g^{\prime},
\end{aligned}
$$

which are all satisfied if only

$$
\begin{aligned}
h & =24, \\
g+16 \nu^{\prime} & =36 \\
g+2 \mu^{\prime} & =12, \\
g+g^{\prime} & =18 \\
\lambda^{\prime} & =-7 .
\end{aligned}
$$

61. If we apply to the same surfaces the reciprocal equation for $\beta$, or, what is the same thing, apply the original equation to the reciprocal surfaces, as given by interchanging the upper and lower halves of the Table of Singularities, we have another series of equations, viz. this is

$$
\begin{aligned}
& 0=54432-54432 \text {, } \\
& 0=27851-27846-h^{\prime}, \\
& 0=18180-18318-g^{\prime}-16 \nu \text {, } \\
& 0=11765-11756-2 h^{\prime}-\lambda \text {, } \\
& h^{\prime} \quad=5 \text {, } \\
& g^{\prime}+16 \nu=-138, \\
& 0=6917-6584-h^{\prime}-g^{\prime}-\mu-8 \nu \text {, } \\
& 2 h^{\prime}+\lambda=9 \text {, } \\
& h^{\prime}+g^{\prime}+\mu+8 \nu=45, \\
& 0=3534-3522-3 h^{\prime}-3 \lambda, \quad 3 h^{\prime}+3 \lambda=12 \text {, } \\
& 0=3024-3144-2 g^{\prime}-16 \nu-g, \quad 2 g^{\prime}+16 \nu+g \quad=-120 \text {, } \\
& 0=1433-1386-2 h^{\prime}-g^{\prime}-\lambda-2 \mu, \quad 2 h^{\prime}+g^{\prime}+\lambda+2 \mu=47 \text {, } \\
& 0=518-504-4 h^{\prime}-6 \lambda, \quad 4 h^{\prime}+6 \lambda=14 \\
& 0=383-322-h^{\prime}-2 g^{\prime}-g-2 \mu, \quad h^{\prime}+2 g^{\prime}+g+2 \mu=61 \text {, } \\
& 0=54 \quad-3 g-3 g^{\prime} \text {, } \\
& 3 g+3 g^{\prime}=54 \text {, }
\end{aligned}
$$


all satisfied if only

$$
\begin{aligned}
& h^{\prime}=5 \text {, } \\
& g^{\prime}+16 \nu=-138, \\
& g^{\prime}+2 \mu=38 \text {, } \\
& g+g^{\prime}=18 \text {, } \\
& \lambda=-1 \text {. }
\end{aligned}
$$

62. I remark however that the cubic scroll XXII or XXIII gives

$$
0=54-(330-272)-2\left(\lambda+\lambda^{\prime}\right),
$$

that is, $\lambda+\lambda^{\prime}=-2$, instead of $\lambda+\lambda^{\prime}=-8$. The investigation is in fact really inapplicable to a scroll, for every point of a scroll has the property of a flecnode; whence if $U=0$ be the equation of the scroll, that of the flecnodal surface is $M . U=0$, containing $U$ as a factor, and there is not any definite curve of intersection constituting the flecnodal curve; but I am nevertheless surprised at the numerical contradiction.

63. Combining the two sets of results, we find

$$
\begin{aligned}
& h=24, \\
& g=g \\
& x=x \\
& \lambda=-1 \\
& \mu=10+\frac{1}{2} g \\
& \nu=-\frac{39}{4}+\frac{1}{16} g \\
& h^{\prime}=5 \\
& g^{\prime}=18-g \\
& x^{\prime}=x^{\prime} \\
& \lambda^{\prime}=-7 \\
& \mu^{\prime}=6-\frac{1}{2} g \\
& \nu^{\prime}=\frac{9}{4}-\frac{1}{16} g
\end{aligned}
$$

and the formula thus is

$$
\begin{aligned}
\beta^{\prime}= & 2 n(n-2)(11 n-24) \\
& -(110 n-272) b+44 q \\
& -(116 n-303) c+\frac{63}{2} r \\
& +\frac{69}{2} \beta+248 \gamma+198 t \\
& -24 C+j-10 \chi+\frac{39}{4} \theta-5 C^{\prime}-18 B^{\prime}-6 \chi^{\prime}-\frac{9}{4} \theta^{\prime} \\
& -x i-x^{\prime} i^{\prime}+\frac{1}{16} g\left(-16 B-8 \chi-\theta+16 B^{\prime}-8 \chi^{\prime}-\theta^{\prime}\right)
\end{aligned}
$$


where $x, x^{\prime}, g$ are constants which remain to be determined. The cubic surfaces fail to determine them, for the reason that in all of them we have $i=0, i^{\prime}=0$; and $16 B+8 \chi+\theta=16 B^{\prime}+8 \chi^{\prime}+\theta^{\prime}$ : this last is a very remarkable relation, for the existence of which $\mathrm{I}$ do not perceive any $\grave{a}$ priori reason.

Substituting herein for $q, r$ their values from No. 11, this may be written in the form

$$
\begin{aligned}
\beta^{\prime}= & 2 n(n-2)(11 n-24)+b(-66 n+184)+c\left(-\frac{369}{4} n+240\right) \\
& +141 \beta+\frac{359}{4} \gamma+66 t \\
& -\quad x^{\prime} i^{\prime}+7 j^{\prime}-6 \chi^{\prime}-\frac{9}{4} \theta^{\prime}-5 C^{\prime}-18 B^{\prime} \\
& -(x+87) i-21 j-\frac{41}{2} \chi+\frac{51}{2} \theta-24 C \\
& +\frac{1}{16} g\left(-16 B-8 \chi-\theta+16 B^{\prime}+8 \chi^{\prime}+\theta^{\prime}\right) .
\end{aligned}
$$

64. We have of course by interchanging the unaccented and accented letters, the reciprocal equation giving the value of $\beta$.

\section{Article Nos. 65 to 68 . Recapitulation.}

65. In recapitulation, I say that we have between the 42 quantities

$$
\begin{aligned}
& n, a, \delta, \kappa ; b, k, t, q, \rho, j ; c, h, r, \sigma, \theta, \chi ; \beta, \gamma, i ; B, C, \\
& n^{\prime}, a^{\prime}, \delta^{\prime}, \kappa^{\prime} ; b^{\prime}, k^{\prime}, t^{\prime}, q^{\prime}, \rho^{\prime}, j^{\prime} ; c^{\prime}, h^{\prime}, r^{\prime}, \sigma^{\prime}, \theta^{\prime}, \chi^{\prime} ; \beta^{\prime}, \gamma^{\prime}, i^{\prime} ; B^{\prime} ; C^{\prime},
\end{aligned}
$$

in all 25 equations, viz. these are

$$
\begin{aligned}
& a=a^{\prime}, \\
& a^{\prime}=n(n-1)-2 b-3 c, \\
& \kappa^{\prime}=3 n(n-2)-6 b-8 c, \\
& \delta^{\prime}=\frac{1}{2} n(n-2)\left(n^{2}-9\right)-\left(n^{2}-n-6\right)(2 b+3 c)+2 b(b-1)+6 b c+\frac{9}{2} c(c-1), \\
& a(n-2)=\kappa-B+\rho+2 \sigma, \\
& b(n-2)=\rho+2 \beta+3 \gamma+3 t, \\
& c(n-2)=2 \sigma+4 \beta+\gamma+\theta, \quad(a b-2 \rho-j)+3(b c-3 \beta-2 \gamma-i), \\
& a(n-2)(n-3)=2(\delta-C)+3(a c-3 \sigma-\chi)+2(a b-2 \rho-j), \\
& b(n-2)(n-3)=4 k+2(b c-3 \beta-2 \gamma-i), \\
& c(n-2)(n-3)=6 h \quad+\quad+3{ }^{\prime}, \\
& q=b^{2}-b-2 k-3 \gamma-6 t, \\
& r=c^{2}-c-2 h-3 \beta, \\
& a=n^{\prime}\left(n^{\prime}-1\right)-2 b^{\prime}-3 c^{\prime}, \\
& \kappa=3 n^{\prime}\left(n^{\prime}-2\right)-6 b^{\prime}-8 c^{\prime}, \\
& \delta=\frac{1}{2} n^{\prime}\left(n^{\prime}-2\right)\left(n^{\prime 2}-9\right)-\left(n^{\prime 2}-n^{\prime}-6\right)\left(2 b^{\prime}+3 c^{\prime}\right)+2 b^{\prime}\left(b^{\prime}-1\right)+6 b^{\prime} c^{\prime}+\frac{9}{2} c^{\prime}\left(c^{\prime}-1\right),
\end{aligned}
$$

C. VI. 


$$
\begin{aligned}
& a^{\prime}\left(n^{\prime}-2\right)=\kappa^{\prime}-B^{\prime}+\rho^{\prime}+2 \sigma^{\prime} \\
& b^{\prime}\left(n^{\prime}-2\right)=\rho^{\prime}+2 \beta^{\prime}+3 \gamma^{\prime}+3 t^{\prime} \\
& c^{\prime}\left(n^{\prime}-2\right)=2 \sigma^{\prime}+4 \beta^{\prime}+\gamma^{\prime}+\theta^{\prime} \\
& a^{\prime}\left(n^{\prime}-2\right)\left(n^{\prime}-3\right)=2\left(\delta^{\prime}-C^{\prime}\right)+3\left(a^{\prime} c^{\prime}-3 \sigma^{\prime}-\chi^{\prime}\right)+2\left(a^{\prime} b^{\prime}-2 \rho^{\prime}-j^{\prime}\right), \\
& b^{\prime}\left(n^{\prime}-2\right)\left(n^{\prime}-3\right)=4 k^{\prime} \quad+\left(a^{\prime} b^{\prime}-2 \rho^{\prime}-j^{\prime}\right)+3\left(b^{\prime} c^{\prime}-3 \beta^{\prime}-2 \gamma^{\prime}-i^{\prime}\right), \\
& c^{\prime}\left(n^{\prime}-2\right)\left(n^{\prime}-3\right)=6 h^{\prime} \quad+\left(a^{\prime} c^{\prime}-3 \sigma^{\prime}-\chi^{\prime}\right)+2\left(b^{\prime} c^{\prime}-3 \beta^{\prime}-2 \gamma^{\prime}-i^{\prime}\right), \\
& q^{\prime}=b^{\prime 2}-b^{\prime}-2 k^{\prime}-3 \gamma^{\prime}-6 t^{\prime}, \\
& r^{\prime}=c^{\prime 2}-c^{\prime}-2 h^{\prime}-3 \beta^{\prime},
\end{aligned}
$$

together with the equations for $\beta$ and $\beta^{\prime}$.

66. The symbols signify as follows; viz.

$n$, order of the surface.

$a$, order of the tangent cone drawn from any point to the surface.

$\delta$, number of nodal edges of the cone.

$\kappa$, number of its cuspidal edges.

$b$, order of nodal curve.

$k$, number of its apparent double points.

$t$, number of its triple points.

$q$, its class.

$\rho$, number of points where nodal curve is met by curve of contact of tangent cone.

$j$, number of pinch-points.

$c$, order of cuspidal curve.

$h$, number of its apparent double points.

$r$, its class.

$\sigma$, number of points where cuspidal curve is met by curve of contact of tangent cone.

$\theta$, number of off-points.

$\chi$, number of close-points.

$\beta$, number of intersections of nodal and cuspidal curves, stationary points on cuspidal curve.

$\gamma$, number of intersections, stationary points on nodal curve.

$i$, number of intersections, not stationary on either curve.

$B$, number of binodes of surface.

$C$, number of cnicnodes.

67. And the accented letters have the like significations in regard to the reciprocal surface; or, referring them to the original surface, we have

$n^{\prime}$, class of the surface.

$a^{\prime}$, class of curve of intersection by any plane.

$\delta^{\prime}$, number of double tangents of curve of intersection. 
$\kappa^{\prime}$, number of its inflexions.

$b^{\prime}$, class of node-couple torse.

$k^{\prime}$, number of its apparent double planes.

$t^{\prime}$, number of its triple planes.

$q^{\prime}$, its order.

$\rho^{\prime}$, order of node-couple curve.

$j^{\prime}$, number of pinch-planes.

$c^{\prime}$, class of spinode torse.

$h^{\prime}$, number of its apparent double planes.

$r^{\prime}$, its order.

$\sigma^{\prime}$, order of spinode curve.

$\theta^{\prime}$, number of off-planes.

$\chi^{\prime}$, number of close-planes.

$\beta^{\prime}$, number of common planes of node-couple and spinode torses, stationary planes of the spinode torse.

$\gamma^{\prime}$, number of common planes, stationary planes of node-couple torse.

$i^{\prime \prime}$, number of common planes, not stationary planes of either torse.

$B^{\prime}$, number of bitropes of surface.

$C^{\prime}$, number of its cnictropes.

68. It is hardly necessary to recall that a spinode plane is a tangent plane meeting the surface in a curve having at the point of contact a spinode or cusp ; the envelope of the spinode planes is the spinode torse, and the locus of their points of contact the spinode curve. And similarly a node-couple plane is a double tangent plane, or plane meeting the surface in a curve having two nodes; the envelope of the planes is the node-couple torse, and the locus of the points of contact the nodecouple curve; the other terms made use of are all explained in the present Memoir.

\section{ADDition, August 3, 1869.}

As in the theory of Curves, so in that of Surfaces, there are certain functions of the order, class, \&c. and singularities which have the same values in the original and the reciprocal figures respectively; for convenience I represent any such identity by means of the symbol $\Sigma$, viz. $\phi(n, a, b, \ldots)=\Sigma$ denotes that the function $\phi(n, a, b, \ldots)$ is equal to the same function $\phi\left(n^{\prime}, a^{\prime}, b^{\prime}, \ldots\right)$ of the accented letters. By what precedes we have $a=\Sigma$; and it is moreover clear that any function of the unaccented letters which is $=0$, or which is equal to a symmetrical function of any of the accented and unaccented letters, or to a function of $a$, is $=\Sigma$; for instance, from the equations of No. 5 we have $3 a^{\prime}-\kappa^{\prime}=3 n-c$, and thence $3 n-c-\kappa=3 a^{\prime}-\kappa-\kappa^{\prime}$, that is, $3 n-c-\kappa=\Sigma$; 
and from one of the equations of No. 11 we have $n-2 C-4 B+\kappa-\sigma-2 j-3 \chi=n+n^{\prime}-a,=\Sigma$; we have thus the system of eight equations,

$$
\begin{aligned}
& \begin{array}{ll}
a & =\Sigma \text {, }
\end{array} \\
& 3 n-c-\kappa=\Sigma \text {, } \\
& a(n-2)-\kappa+B-\rho-2 \sigma=\Sigma \text {, } \\
& b(n-2)-\rho-2 \beta-3 \gamma-3 t=\Sigma \text {, } \\
& c(n-2)-2 \sigma-4 \beta-\gamma-\theta=\Sigma \text {, } \\
& n+\kappa-\sigma-2 C-4 B-2 j-3 \chi=\Sigma \text {, } \\
& 2 q-2 \rho+\beta+3 i+j=\Sigma \text {, } \\
& 3 r+c-5 \sigma-\beta-4 \theta+2 i+\chi=\Sigma \text {; }
\end{aligned}
$$

or if from these we eliminate $\kappa, \rho, \sigma$, then the system of five equations,

$$
\begin{array}{lr}
a & =\Sigma, \\
n(c-8)-4 \beta-\gamma-\theta+4 C+8 B+6 \chi+4 j & =\Sigma, \\
(a-b)(n-2)-11 n+3 c+4 C+9 B+2 \beta+3 \gamma+3 t+6 \chi+4 j=\Sigma, & =\Sigma, \\
3 r-20 n+6 c-\beta+2 i+10 C+20 B+16 \chi+10 j-4 \theta & =\Sigma . \\
2 q-2 b(n-2)+5 \beta+6 \gamma+6 t+3 i+j &
\end{array}
$$

By means of a theorem of Dr Clebsch's I was led to the following expression for the "deficiency" of a surface of the order $n$ having the singularities considered in the foregoing Memoir:

Deficiency $=\frac{1}{6}(n-1)(n-2)(n-3)-(n-3)(b+c)+\frac{1}{2}(q+r)+2 t+\frac{7}{2} \beta+\frac{5}{2} \gamma+i-\frac{1}{8} \theta$. This should be equal to the deficiency of the reciprocal surface, viz. we must have

$$
2(n-1)(n-2)(n-3)-12(n-3)(b+c)+6 q+6 r+24 t+42 \beta+30 \gamma+12 i-\frac{3}{8} \theta=\Sigma ;
$$

but from a combination of the last-mentioned five equations we have

$$
\begin{aligned}
-2 n^{3}+6 n^{2}+4 n+(12 n-36) b & +(12 n-48) c-6 q-6 r-24 t \\
& -41 \beta-30 \gamma-13 i-7 j-8 \chi+2 \theta-4 C-10 B=\Sigma ;
\end{aligned}
$$

and adding to the last preceding equation we have

$$
26 n-12 c+\beta-i-7 j-8 \chi+\frac{1}{2} \theta-4 C-10 B=\Sigma .
$$

Substituting for $\Sigma$ its value in terms of the accented letters, we obtain for $\beta^{\prime}$ the value

We have

$$
\begin{array}{r}
\beta^{\prime}=\beta+26 n-12 c+i^{\prime}+7 j^{\prime}+8 \chi^{\prime}-\frac{1}{2} \theta^{\prime}+4 C^{\prime}+10 B^{\prime} \\
-26 n^{\prime}+12 c^{\prime}-i-7 j-8 \chi+\frac{1}{2} \theta-4 C-10 B .
\end{array}
$$

and thence

$$
c^{\prime}=-3 a+\kappa+3 n^{\prime},
$$

$$
12 c^{\prime}-26 n^{\prime}=-36 a+12 \kappa+10 n^{\prime}
$$


writing herein

the value is

$$
n^{\prime}=a+\kappa-\sigma-2 C-4 B-2 j-3 \chi-\sigma,
$$

$$
=-26 a+22 \kappa-20 C-40 B-20 j-30 \chi-10 \sigma .
$$

Substituting for $\kappa$ its value $=a(n-2)+B-\rho-2 \sigma$, we have

$$
12 c^{\prime}-26 n^{\prime}=a(22 n-70)-20 C-18 B-20 j-30 \chi-22 \rho-44 \sigma ;
$$

or substituting for $a, \rho, \sigma$ their values, this is

$$
\begin{aligned}
= & \{n(n-1)-2 b-3 c\}(22 n-70)-20 C-18 B-20 j-30 \chi \\
& -22 b(n-2)+44 \beta+66 \gamma+66 t \\
& -27 c(n-2)+108 \beta+27 \gamma+27 \theta,
\end{aligned}
$$

and adding hereto the remaining terms,

$$
\begin{array}{r}
\beta+26 n-12 c+i^{\prime}+7 j^{\prime}+8 \chi^{\prime}-\frac{1}{2} \theta^{\prime}+4 C^{\prime}+10 B^{\prime} \\
-i-7 j-8 \chi+\frac{1}{2} \theta-4 C-10 B
\end{array}
$$

we have

$$
\begin{gathered}
\beta^{\prime}=2 n(n-2)(11 n-24)+b(-66 n+184)+c(-93 n+252)+153 \beta+93 \gamma+66 t \\
+i^{\prime}+7 j^{\prime}+8 \chi^{\prime}-\frac{1}{2} \theta^{\prime}+4 C^{\prime}+10 B^{\prime} \\
-i-27 j-38 \chi+\frac{55}{2} \theta-24 C-28 B .
\end{gathered}
$$
have

Comparing this with the value of $\beta^{\prime}$, No. 63 of the foregoing Memoir, we should

$$
\begin{aligned}
0= & \frac{13}{4} c n-12 c-12 \beta-\frac{13}{4} \gamma \\
& -\left(x^{\prime}+1\right) i^{\prime}-14 \chi^{\prime}-\frac{7}{4} \theta^{\prime}-9 C^{\prime}-28 B^{\prime} \\
& -(x+86) i+6 j+\frac{35}{2} \chi-2 \theta+28 B \\
& +\frac{1}{16} g\left(-16 B-8 \chi-\theta+16 B^{\prime}+8 \chi^{\prime}+\theta^{\prime}\right),
\end{aligned}
$$

or, what is the same thing,

if for shortness

$$
0=13 c n-48 c-48 \beta-13 \gamma+\Phi,
$$

$$
\begin{aligned}
\Phi= & -\left(4 x^{\prime}+4\right) i^{\prime}-56 \chi^{\prime}-7 \theta^{\prime}-36 C^{\prime}-112 B^{\prime} \\
& -(4 x+344) i+24 j+70 \chi-8 \theta+112 B \\
& +\frac{1}{4} g\left(-16 B-8 \chi-\theta+16 B^{\prime}+8 \chi^{\prime}+\theta^{\prime}\right) .
\end{aligned}
$$

I do not attempt to verify this equation, but I will partially verify a result deducible from it; viz. if $\Phi^{\prime}$ is the like function of the accented letters, then we have

where

$$
\Phi-\Phi^{\prime}=\Pi-\Pi^{\prime}
$$

$$
\begin{array}{r}
\Pi=\left(4 x^{\prime}-4 x-340\right) i+24 j+126 \chi+224 B+36 C-\theta \\
-\frac{1}{2} g(16 B+8 \chi+\theta)
\end{array}
$$


and $\Pi^{\prime}$ is the like function of the accented letters. And this being so, we should have

$$
13 c n-48 c-48 B-13 \gamma+\Pi=13 c^{\prime} n^{\prime}-48 c^{\prime}-48 \beta^{\prime}-13 \gamma^{\prime}+\Pi^{\prime},
$$

or, as this may be written,

$$
13 c n-48 c-48 \beta-13 \gamma+\Pi=\Sigma \text {. }
$$

We have

$$
26 n-12 c+\beta-i-7 j-8 \chi+\frac{1}{2} \theta-4 C-10 B=\Sigma ;
$$

and multiplying by -4 and adding, the equation to be verified is

$$
13 n(c-8)-13(4 \beta+\gamma)+\Pi+4 i+28 j+32 \chi-2 \theta+16 C+40 B=\Sigma .
$$

But we have from the Memoir

$$
-13 n(c-8)+13(4 \beta+\gamma) \quad-52 j-78 \chi+13 \theta-52 C-104 B=\Sigma,
$$

which reduces the equation to

$$
\Pi+4 i-24 j-46 \chi+11 \theta-36 C-64 B=\Sigma ;
$$

or substituting for $\Pi$ its value, this is

that is

$$
\left(4 x^{\prime}-4 x-336\right) i+80 \chi+10 \theta+160 B-2 \lambda(16 B+8 \chi+\theta)=\Sigma,
$$

$$
4\left(x^{\prime}-x-84\right) i-\left(\frac{1}{2} g-10\right)(16 B+8 \chi+\theta)=\Sigma,
$$

an equation which is satisfied if

and

$$
i^{\prime}=i, \quad x^{\prime}=x,
$$

$$
g=20 \text {, or else } 16 B+8 \chi+\theta=16 B^{\prime}+8 \chi^{\prime}+\theta^{\prime} .
$$

